\title{
Produção científica dos cursos de pós-graduação em Saúde Coletiva no período 1998-2006
}

\author{
Scientific production of Brazilian graduate courses \\ in Public Health during 1998-2006
}

Francisco Viacava $^{1}$

${ }^{1}$ Departamento de
Informações Em Saúde,
Centro deInformação
Científica eTecnológica,
Fundação Oswaldo Cruz.
Av. Brasil 4365,
M anguinhos. 21045-900
Rio deJaneiro RJ.
viacava@icict.fiocruz.br
Abstract The massive growth of graduate programs in Brazil, observed in the areas of Health Sciences and Public H ealth, makes it necessary to consider the processes underlying the diffusion of science. Qualitative analyses on the main themes of the field have been conveyed using scientific journals and a directory of research groups. The agreement made between ABRASCO and CAPES opened the possibility of using a database that, although construed for the evaluation of graduate programs, may be useful for different purposes. In this study, the full papers registered in three triennials from 1998 to 2006 were analyzed. In order to accomplish this it was necessary to standardize the titles of the scientific journals and also the titles of full text articles published, in large part becausethe co-authorship of researchers from different programs resulted in duplications of entries. O nly then was possible to build a database where every article is registered no more than once. From this point, the changes throughout the three periods were analyzed, as well as the most frequently consulted journals, number of authors, language, national and foreign journals, and regional and interregional cooperation. The data presented here may be useful for the discussions on the scientific production and dissemination of knowledge in the area of Public $\mathrm{H}$ ealth. Key words Scientific production, Public health, Graduate programs
Resumo 0 grandecrescimento da pós-graduação no Brasil, também observado na grande área de Saúdee na Saúde Coletiva, torna necessário equacionar como se processa a difusão científica. Análises qualitativas sobre as principais temáti cas têm sido feitas a partir das revistas científicas e do diretório de grupos de pesquisa. 0 acordo feito entre a Abrasco e a CAPES abriu a possibilidade de exploração deum banco de dados construído eusado para a avaliação dos programas de pós-graduação, sob outras perspectivas. N essetrabalho, foram analisados os artigos completos registrados na basenos três triênios do período 1998 - 2006. Para tanto, fez-se uma padronização dos títulos dos periódi cos e dos títulos dos artigos completos publicados em periódicos, já que havia duplicações, em grande parte devidasà coautoria de docentes de diferentes programas. Com isso, foi possível construir um banco de dados no qual cada artigo é registrado uma única vez, a partir do qual foi possível analisar a evolução ao longo dos triênios dos periódicos mais frequentemente usados, número de autores, idioma, nacionalidade das revistas e cooperação interregional. Os dados aqui apresentados poderão subsidiar as discussões sobre a produção científica e difusão do conhecimento na área. Palavras-chave Produção científica, Saúde Cole tiva, Pós-graduação 
Introdução

A análise da produção científica dos cursos de pós-graduação da área de Saúde Coletiva assume especial relevância quando se leva em conta a natureza dessa área enquanto um campo de aplicação das ciências biológicas e das ciências sociais/humanas. N esse sentido, como qualquer outro campo do conhecimento científico, a produção se faz dentro das regras do método científico, mas, além disso, está fortemente direcionada para a intervenção, seja do ponto de vista do entendimento do processo saúde/doença ou da avaliação do alcance das políticas de saúde, e das condições de vida e saúde da população. Estudos recentes têm evidenciado a estreita vinculação entre os saberes e as práticas nas três grandes áreas temáticas da Saúde Coletiva: planejamento e gestão ${ }^{1}$, ciências sociais ${ }^{2}$ e epidemiologi ${ }^{3}$.

Segundo Barata4, nos últimos dez anos, o número de programas de mestrado e doutorado cresceu de maneira mais acentuada do que no conjunto detodas as áreas de conhecimento, che gando em 2008 com 47 programas, dos quais 21 têm doutorado. Ainda que fortemente concentrados na Região Sudeste, os programas estão distribuídos por todas as regiões, com exceção da Região N orte.

Do ponto de vista da difusão da produção científica, há poucos estudos publicados entre os quais se incluem uma avaliação dos artigos publicados pelos cursos de pós-graduação entre 1990 e $1995^{5}$, estudos sobre artigos publicados em principais revistas da área ${ }^{6,7}$, e uma análise sobre a produção científica dos cursos de pósgraduação no período 2000-2004', com ênfase no fator de impacto das principais revistas usadas pelos pesquisadores da área para divulgação da produção científica.

Neste artigo, analisam-se as principais características do processo de produção científica da área de Saúde Coletiva e as formas de difusão desse conhecimento no âmbito da comunidade científica tal como cadastrada na base de dados do sistema COLETA da CAPES para o período 1998-2006.

\section{Material emétodo}

0 enorme crescimento da pós-graduação no Brasil ${ }^{9-11}$, também evidenciado na área de Saúde Coletiva ${ }^{48}$, e a decisão de se analisar os últimos dez anos de atividades, tornou imperativo um recortedo tipo de produção científica. Assim sen- do, a análise foi circunscrita aos artigos publicados em periódicos, principal forma de difusão dessa produção. Também foi necessário abrir mão de alguns objetivos propostos originalmente no projeto, já que o banco de dados do sistema COLETA ainda se ressente de problemas de incompletude de vários campos imprescindíveis para uma definição das áreas temáticas, como, por exemplo, palavras-chave. A análise dos títulos para definição de áreas temáticas também se mostrou inviável, já que se tratava de analisar mais de 11,5 mil artigos.

A análise das publicações geradas no âmbito dos cursos de pós-graduação de Saúde Coletiva foi feita usando-se as informações existentes no sistema COLETA da CAPES, considerando-se 0 período de 1998 a 2006. Os dados foram cedidos pela CAPES separadamente para cada tema e, no caso dos artigos publicados em periódicos, incluem também o ano de 2007.

0 arquivo contendo artigos em periódicos apresentava 16.423 registros. Foram incluídos na análise apenas os "trabal hos completos", excluindo-se os resumos em periódicos e outras formas de produção bibliográfica ( $n=3.192)$. Dada a existência de duplicação de registros, foi necessário realizar uma crítica do banco de dados de tal forma que cada artigo fosse contabilizado uma única vez (Figura 1). Para tanto, os títulos dos artigos foram padronizados eliminando-se todo tipo de acentuação, de modo que as duplicações pudessem ser mais facilmente evidenciadas. Uma vez identificada a duplicação do título, procedeu-se à verificação do ano de indexação e dos programas nos quais 0 artigo foi indexado, posto que quando é de autoria de docentes que pertencem a programas diferentes ele é registrado por cada um dos programas. Quando o mesmo título foi registrado em anos diferentes por cada programa, foi realizada uma busca na WEB para recuperar 0 artigo no formato publicado e considerou-secomo ano base da produção 0 ano do periódico. Em 61 desses casos, não foi possível localizar a publicação etais textos estão excluídos na análise. Foram identificadas 292 duplicações dos seguintes tipos:

(a) Artigos registrados mais de uma vez em um mesmo programa no mesmo ano: nesse caso consi derou-se que havia uma duplicação e manteve-se apenas um registro na base;

(b) Artigos registrados em um mesmo programa em anos diferentes: também foi considerada duplicação; manteve-se o registro no ano base correspondente ao da publicação e excluíram-se os demais; 


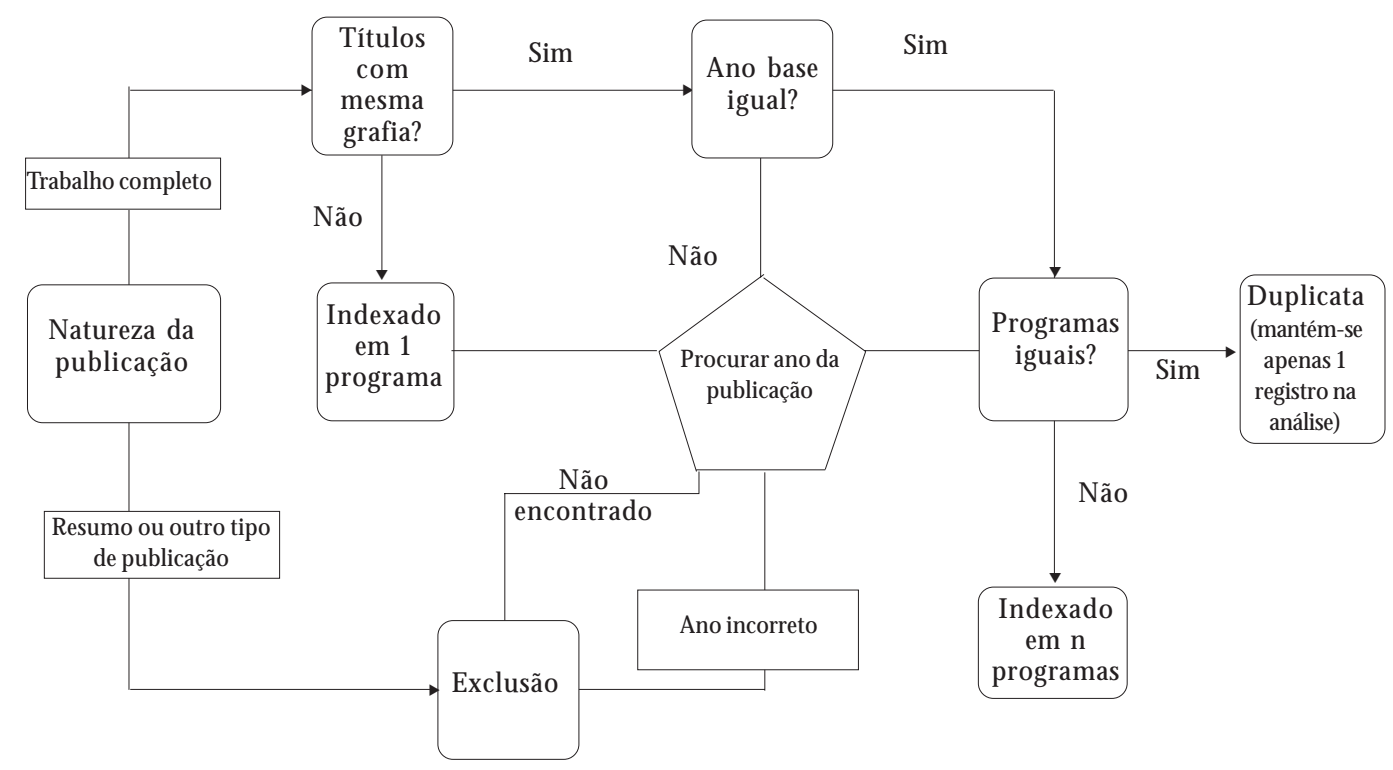

Figura 1. Processo de crítica da indexação dos registros de artigos em periódicos.

(c) Artigos registrados no mesmo ano em diversos programas: os registros forma mantidos em todos os programas onde estavam registrados;

(d) Artigos registrados em diversos programas em anos diferentes: os registros foram mantidos em todos os programas, mas o ano base foi corrigido considerando-se o da publicação.

Feitas as correções necessárias, passou-se a contar com um total de 11.671 artigos publicados, no período 1998-2007, por pelo menos um programa da área de Saúde Coletiva. Em resumo, para a análise dos dados, foram excluídos $23,3 \%$ dos registros correspondentes a resumos e outras publicações $(21 \%)$, duplicações $(1,9 \%)$ e referências que não foram encontradas $(0,4 \%)$ (Tabela 1). Ainda assim, permanecem duplicados na base os artigos registrados com grafias diferentes, que só poderiam ser identificados por um processo de leitura caso a caso.

Uma vez identificadas as duplicações, como já mencionado, foram construídos dois bancos de dados: com esem duplicações. 0 banco sem duplicações foi usado para analisar a evolução da produção ao longo do período 1998-2007 e suas características em termos de número de autores, idioma de redação e principais periódicos de divulgação.
Tabela 1. Número de publicações segundo a indexação na base da CAPES.

\begin{tabular}{lrr}
\hline \multicolumn{1}{c}{ Indexação } & N & \multicolumn{2}{c}{$\%$} \\
\hline Duplicatas & 303 & 2,0 \\
Artigo completo indexado em 1 programa & 10604 & 69,6 \\
Artigo completo indexado em 2 programas & 948 & 6,2 \\
Artigo completo indexado em 3 programas & 109 & 0,7 \\
Artigo completo indexado em 4 programas & 10 & 0,1 \\
Referência não encontrada & 61 & 0,4 \\
Resumos e outras publicações & 3192 & 21,0 \\
Total & 15227 & 100 \\
\hline
\end{tabular}

Para análise dos temas abordados, fez-seuma classificação dos principais periódicos, tendo em vista as restrições já apontadas. A análise do número de artigos por docente e por programa levou em conta as duplicações, já que parte dos artigos foi elaborada por docentes de mais de um programa. N esse caso, foi excluído 0 ano de 2007, uma vez que não existia informação sobre docentes. A base de artigos indexados em mais de um programa também foi utilizada para verificar as cooperações intra e interregionais entre 
docentes. Todas as análises foram feitas considerando os três triênios avaliados pela CAPES, ou seja, 1998-2000, 2001-2003 e2004-2006.

\section{Resultados}

Evolução do número de artigos no período 1998-2007

A composição percentual das publicações científicas na área deSaúde Coletivaindica que, ao longo dos nove anos de observação, quase $60 \%$ das publicações ocorrerem sob forma de artigo em periódico (Gráfico 1). Entretanto, no último triênio, houve uma ligeira diminuição da proporção dos artigos em periódicos em relação ao aumento da participação em apresentações em congressos e livros e capítulos de livros.

Quanto ao número de artigos, ressalta-se em primeiro lugar um enorme crescimento (cerca de vinte vezes) ao longo da década, passando de cerca de menos de 591 em 1998 para 11.671 artigos em 2007. No Gráfico 2, é possível verificar que, depois de um período de crescimento no começo da série histórica (1998-2000), segue-se certa estagnação (em torno de mil artigos por ano) até 2002. A partir de 2003, a tendência é de crescimento a taxas cada vez maiores até 2007. No último biênio (2006/2007), verifica-se que essa taxa crescimento chegou a $32 \%$.
Evolução do número de artigos por programa e por docente

Para analisar a produção de artigos em periódicos por programa, segundo o número de docentes de cada programa, partiu-se do conjunto de artigos queforam indexados em um ou mais programas no período 1998-2006. Considerando que esses podem ter sido escritos por autores pertencentes a programas diferentes, foi necessário trabal har com duplicações de artigos

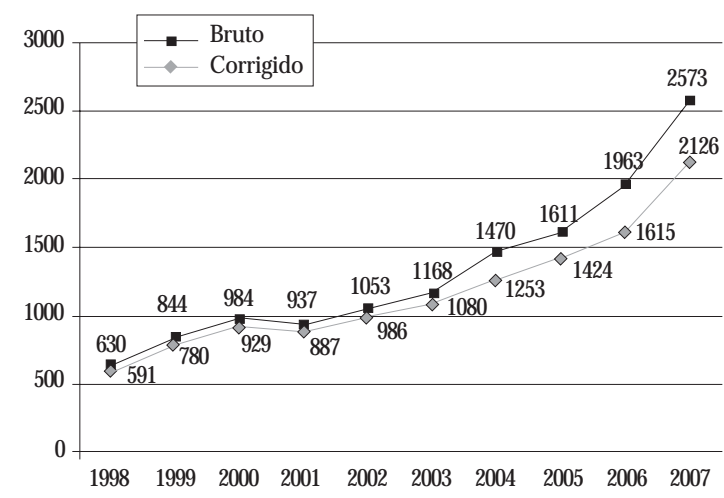

Gráfico 2. Número corrigido de artigos em periódicos indexados no COLETA: 1998-2007.

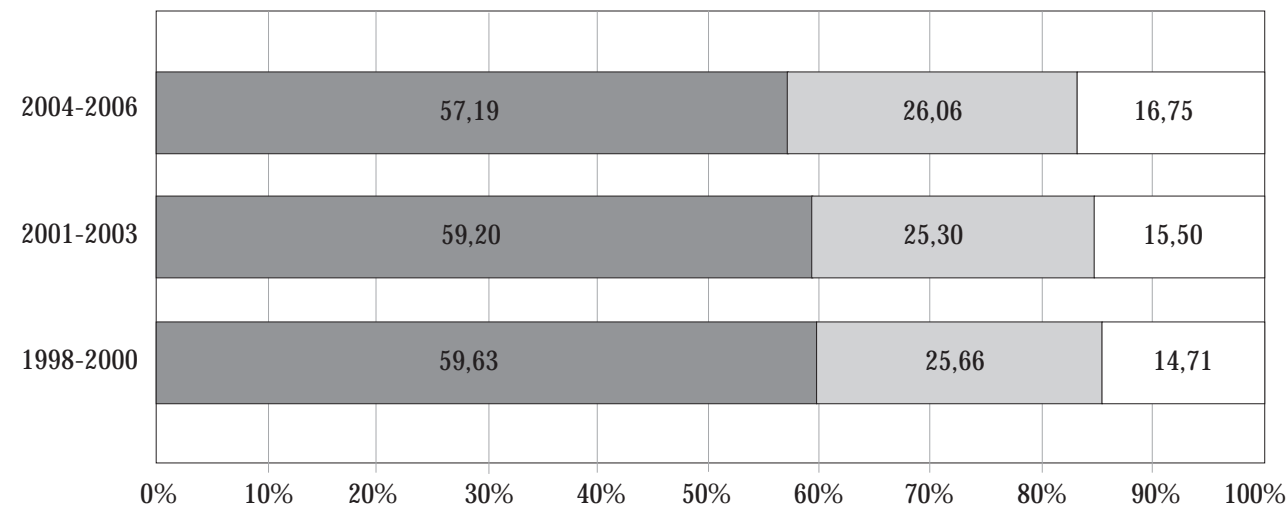

Artigos completos publicados $\square$ Livros e capítulos em livros $\square$ Trabalho em anais

Gráfico 1. Divulgação da produção científica na área de Saúde Coletiva segundo triênios e formato da publicação. 
( $n=10.344)$, contabilizados nos respectivos programas. Os dados foram obtidos separadamente para cada triênio, de tal forma que fosse possível fazer uma apreciação da evolução do indicador ao longo do período 1998-2006. Cabe esclarecer que 0 indicador foi construído usando como denominador o número total de docentes e não apenas os docentes permanentes, já que para o primeiro triênio não seria possível fazer a distinção. Pelo mesmo motivo, o número de artigos não foi corrigido pelos Q ualis das revistas. O s val ores devem ser vistos apenas como indicativos da maior ou menor produção entre cursos e ao longo do período analisado (Tabela 2).

Tabela 2. Número de artigos por docente segundo programas e triênio de avaliação.

\begin{tabular}{|c|c|c|c|c|c|c|c|c|c|c|c|c|c|}
\hline \multirow[t]{3}{*}{ Sigla do IES } & \multirow[t]{3}{*}{ Nível } & \multicolumn{4}{|c|}{ Artigos } & \multicolumn{4}{|c|}{ Docentes } & \multicolumn{4}{|c|}{ Artigos/docentes/triênio } \\
\hline & & \multicolumn{3}{|c|}{ Triênio } & \multirow{2}{*}{ Total } & \multicolumn{3}{|c|}{ Triênio } & \multirow{2}{*}{ Total } & \multicolumn{3}{|c|}{ Triênio } & \multirow[t]{2}{*}{ Total } \\
\hline & & 1 & 2 & 3 & & 1 & 2 & 3 & & 1 & 2 & 3 & \\
\hline UFMA & M A & 47 & 0 & 0 & 47 & 47 & 0 & 0 & 47 & 1 & - & - & - \\
\hline UFC & M A & 48 & 60 & 91 & 199 & 46 & 39 & 41 & 126 & 1 & 1,5 & 2,2 & 1,6 \\
\hline UNIFOR & M A & 0 & 45 & 79 & 124 & 0 & 17 & 40 & 57 & 0 & 2,6 & 2 & 2,2 \\
\hline UECE & M A & 47 & 71 & 92 & 210 & 43 & 32 & 49 & 124 & 1,1 & 2,2 & 1,9 & 1,7 \\
\hline UFPE & MA & 36 & 22 & 28 & 86 & 17 & 23 & 39 & 79 & 2,1 & 1 & 0,7 & 1,1 \\
\hline FESP/UPE & $M P$ & 0 & 0 & 20 & 20 & 0 & 0 & 18 & 18 & - & - & 1,1 & 1,1 \\
\hline CPqAM & $\mathrm{D}$ & 82 & 126 & 157 & 365 & 58 & 64 & 98 & 220 & 1,4 & 2 & 1,6 & 1,7 \\
\hline CPqAM & M P & 0 & 0 & 65 & 65 & 0 & 0 & 44 & 44 & - & - & 1,5 & 1,5 \\
\hline UFBA & $\mathrm{D}$ & 79 & 141 & 197 & 417 & 57 & 74 & 107 & 238 & 1,4 & 1,9 & 1,8 & 1,8 \\
\hline UFBA & $M P$ & 0 & 0 & 38 & 38 & 0 & 0 & 84 & 84 & - & - & 0,5 & 0,5 \\
\hline UEFS & M A & 7 & 0 & 0 & 7 & 70 & 0 & 0 & 70 & 0,1 & - & - & 0,1 \\
\hline UEFS & $M A$ & 0 & 0 & 126 & 126 & 0 & 0 & 61 & 61 & - & - & 2,1 & 2,1 \\
\hline UFES & M A & 0 & 23 & 41 & 64 & 0 & 17 & 51 & 68 & - & 1,4 & 0,8 & 0,9 \\
\hline UFRJ & M A & 62 & 136 & 123 & 321 & 44 & 77 & 60 & 181 & 1,4 & 1,8 & 2,1 & 1,8 \\
\hline UERJ & $\mathrm{D}$ & 312 & 291 & 359 & 962 & 122 & 143 & 148 & 413 & 2,6 & 2 & 2,4 & 2,3 \\
\hline FIOCRUZ & $D$ & 509 & 627 & 777 & 1913 & 245 & 264 & 399 & 908 & 2,1 & 2,4 & 1,9 & 2,1 \\
\hline FIOCRUZ & $\mathrm{D}$ & 94 & 114 & 178 & 386 & 55 & 86 & 100 & 241 & 1,7 & 1,3 & 1,8 & 1,6 \\
\hline FIOCRUZ & M P & 0 & 0 & 290 & 290 & 0 & 0 & 186 & 186 & - & - & 1,6 & 1,6 \\
\hline FIOCRUZ & M P & 0 & 0 & 85 & 85 & 0 & 0 & 54 & 54 & - & - & 1,6 & 1,6 \\
\hline FIOCRUZ & $\mathrm{D}$ & 0 & 0 & 47 & 47 & 0 & 0 & 22 & 22 & - & - & 2,1 & 2,1 \\
\hline UNESA & MP & 0 & 0 & 21 & 21 & 0 & 0 & 31 & 31 & - & - & 0,7 & 0,7 \\
\hline UFM G & $\mathrm{D}$ & 42 & 114 & 141 & 297 & 34 & 49 & 57 & 140 & 1,2 & 2,3 & 2,5 & 2,1 \\
\hline USP/FM & $D$ & 88 & 110 & 148 & 346 & 50 & 53 & 55 & 158 & 1,8 & 2,1 & 2,7 & 2,2 \\
\hline USP/FSP & $D$ & 371 & 479 & 630 & 1480 & 347 & 309 & 348 & 1004 & 1,1 & 1,6 & 1,8 & 1,5 \\
\hline USP/RP & $D$ & 45 & 40 & 110 & 195 & 35 & 36 & 45 & 116 & 1,3 & 1,1 & 2,4 & 1,7 \\
\hline UNICAMP & $\mathrm{D}$ & 76 & 100 & 136 & 312 & 84 & 71 & 86 & 241 & 0,9 & 1,4 & 1,6 & 1,3 \\
\hline UNESP/BOT & M A & 42 & 49 & 119 & 210 & 35 & 45 & 71 & 151 & 1,2 & 1,1 & 1,7 & 1,4 \\
\hline UNIFESP & $\mathrm{MA}$ & 33 & 82 & 27 & 142 & 23 & 33 & 21 & 77 & 1,4 & 2,5 & 1,3 & 1,8 \\
\hline UNIFESP & $\mathrm{D}$ & 0 & 0 & 33 & 33 & 0 & 0 & 16 & 16 & - & - & 2,1 & 2,1 \\
\hline FCMSCSP & MP & 0 & 0 & 66 & 66 & 0 & 0 & 42 & 42 & - & - & 1,6 & 1,6 \\
\hline UNISANTOS & M A & 0 & 0 & 56 & 56 & 0 & 0 & 40 & 40 & - & - & 1,4 & 1,4 \\
\hline UEL & M A & 39 & 37 & 48 & 124 & 28 & 26 & 35 & 89 & 1,4 & 1,4 & 1,4 & 1,4 \\
\hline UFSC & $\mathrm{MA}$ & 37 & 46 & 103 & 186 & 40 & 33 & 46 & 119 & 0,9 & 1,4 & 2,2 & 1,6 \\
\hline UFRGS & $\mathrm{D}$ & 27 & 115 & 120 & 262 & 17 & 48 & 62 & 127 & 1,6 & 2,4 & 1,9 & 2,1 \\
\hline UFPEL & $\mathrm{D}$ & 99 & 114 & 186 & 399 & 28 & 27 & 34 & 89 & 3,5 & 4,2 & 5,5 & 4,5 \\
\hline UNISINOS & M A & 0 & 35 & 81 & 116 & 0 & 24 & 31 & 55 & - & 1,5 & 2,6 & 2,1 \\
\hline ULBRA & M A & 0 & 56 & 79 & 135 & 0 & 33 & 44 & 77 & - & 1,7 & 1,8 & 1,8 \\
\hline UFMT & M A & 98 & 0 & 0 & 98 & 81 & 0 & 0 & 81 & 1,2 & - & - & 1,2 \\
\hline UFMT & $\mathrm{MA}$ & 0 & 9 & 34 & 43 & 0 & 13 & 51 & 64 & - & 0,7 & 0,7 & 0,7 \\
\hline UFMS & M A & 22 & 28 & 0 & 50 & 30 & 41 & 0 & 71 & 0,7 & 0,7 & - & 0,7 \\
\hline TOTAL & & 2.342 & 3.070 & 4.932 & 10.344 & 1.636 & 1.677 & 2.716 & 6.029 & 1,4 & 1,8 & 1,8 & 1,7 \\
\hline
\end{tabular}

Obs.: M A: M estrado acadêmico; M P: M estrado profissional; D: Doutorado (e M estrado). 
É importante verificar que os programas se diferenciam enormemente quanto ao volume da produção científica e quanto aos indicadores de produtividade científica. Pela evolução do indicador artigo/docente/triênio, nota-se quea produtividade média foi de 1,4 artigos/docente para o conjunto dos programas no primeiro triênio, passando para 1,8 no segundo triênio, mantendo-senessa magnitudeno terceiro triênio. Entre tanto, outras medidas de tendência central (me dianas) mostram que houve uma melhora gradual da produtividade por docente ao longo do período (Tabela 3).

\section{Periódicos mais utilizados}

Para verificar os periódicos mais utilizados pela área de Saúde Coletiva na difusão da produção científica, também foi necessário realizar inicialmente uma crítica na base de dados para padronizar ostítulos dos periódicos. Assim, como já apontado em 1997, a exemplo do que ocorria com o sistema EXECAPES 5 , a entrada de dados no sistema COLETA também não utiliza críticas ou títulos padronizados, como acontece, por exemplo, na plataforma Lattes do CN Pq. Por esse motivo, foi necessário analisar todos os títulos escritos nos mais diversos formatos e definir apenas um formato para cada título.

Considerando apenas os trabalhos comple tos, publicados nos dez anos compreendidos entre 1998 e2006, verifica-seque, após a padronização, dos 3.502 títulos existentes no banco original daCAPES, cerca de $25 \%$ correspondiam aosmesmos títulos e estavam escritos de diversas maneiras. D eve-semencionar também que, nessa análise, foram mantidos os títulos atuais dos periódicos para os quais foram contabilizados também títulos anteriores como o caso da Revista Panamericana deSaúdePública, antigo Boletim daOPS, e a Revista Epidemiologia e Serviços de Saúde, antigo Informe Epidemiológico do SUS.

Tabela 3. Artigos publicados em periódicos por docente por triênio.

\begin{tabular}{lrrcrc}
\hline & \multicolumn{3}{c}{ Triênio } & & Total \\
\cline { 2 - 4 } & $1998-2000$ & $2001-2003$ & $2004-2006$ & & 1998-2006 \\
\hline Cursos & 24 & 26 & 36 & \\
M ediana & 1,35 & 1,65 & 1,8 & & 1,6 \\
Mínimo & 0,1 & 0,7 & 0,5 & & 0,1 \\
Máximo & 3,5 & 4,2 & 5,5 & & 4,5 \\
\hline
\end{tabular}

Ao longo do período analisado, verifica-se que o número de títulos usados para divulgação da produção aumentou em cerca de $20 \%$ por triênio, enquanto o número de artigos teve um crescimento de $28 \%$ do primeiro para o segundo triênio e de $46 \%$ do segundo para o terceiro.

A análise dos títulos dos periódicos indica que, para os três períodos compreendidos entre 1998 e 2006, o conjunto dos programas publicou em média quinze artigos por triênio em 24 periódicos (Tabela 4). As três revistas mais utilizadas foram: Cadernos de Saúde Pública (12,1\%), Revista de Saúde Pública (6,3\%) e Ciência \& Saúde Coletiva (4,7\%). A participação das três revistas ao longo do período foi de $23 \%$ para o primeiro triênio, $21 \%$ para o segundo e $25 \%$ para o terceiro. Entretanto, quando se observa o universo de revistas, registradas no COLETA ( $\mathrm{N}=2153)$, torna-se evidente que novos títulos vêm sendo incorporados a taxas próximas de $20 \%$ por triênio, ressaltando maior diversificação nos últimos anos da série. Quando se analisam as revistas que apresentaram no mínimo quinze publicações em pelo menos um triênio, verifica-seque apenas 42 títulos foram usados para divulgar cerca de $50 \%$ da produção no último triênio.

\section{Temas pesquisados}

Para analisar os temas pesquisados pela área ao longo da década, classificaram-se as revistas mais procuradas pelos autores (pelo menos quinze artigos em um dos três períodos) segundo campos temáticos. Como não existe uma classificação padronizada, fez-se aqui uma primeira categorização usando-se informações da própria revista (Tabela 5).

Partindo dessa classificação, verifica quehouve uma diminuição relativa de artigos publicados em revistas de saúde pública e aumento da proporção de artigos publicados em revistas com temáticas mais específicas (Gráfico 3). Ao longo dos triênios, há indícios de aumento da participação da produção em revistas das áreas clínicas edas revistas cujo enfoque principal éa atenção à saúde e diminuição das revistas classificadas como de ciências sociais. Esseachado mereceuma maior exploração que deve incluir uma revisão da classificação utilizada.

Qualis das revistas

Outro aspecto explorado nessa análise foi a participação relativa de revistas segundo os Qual is no período 2001-2006. Para tanto, foram anali- 
sados apenas os programas que estavam em atividade desde2001. Verifica-se no Gráfico 4, comparando-se a evolução nos dois triênios, que houve um expressivo aumento da publicação de artigos em revistas IA e, em menor importância, das revistas IC, NA e NC. N ota-se também uma redução da importância das revistas NB elocais.
Evolução do número de artigos por idioma de publicação

No que se refere ao idioma dos artigos, observa-se um padrão constante ao longo dos três triênios analisados, sendo quecerca de $70 \%$ deles são publicados em português e quase $30 \%$, em

Tabela 4. Relação de títulos dos periódicos nos quais foram publicados pelo menos quinze artigos em um dos três triênios.

\begin{tabular}{|c|c|c|c|c|c|c|c|c|c|}
\hline \multirow[b]{2}{*}{ Títulos } & \multicolumn{3}{|c|}{$1998-2000$} & \multicolumn{3}{|c|}{ 2001-2003 } & \multicolumn{3}{|c|}{ 2004-2006 } \\
\hline & $\mathrm{n}$ & $\%$ & $\% a c$ & $\mathrm{n}$ & $\%$ & $\% a c$ & $\mathrm{n}$ & $\%$ & $\% a c$ \\
\hline Cadernos de Saúde Pública & 226 & 9,8 & 9,8 & 361 & 12 & 12,2 & 569 & 13,3 & 13,3 \\
\hline Revista de Saúde Pública & 156 & 6,8 & 16,6 & 168 & 5,7 & 17,9 & 281 & 6,5 & 19,8 \\
\hline Ciência \& Saúde Coletiva & 144 & 6,3 & 22,9 & 91 & 3,1 & 21,0 & 219 & 5,1 & 24,9 \\
\hline Série Estudos em Saúde Coletiva & 63 & 2,7 & 25,6 & 0 & 0 & 21,0 & 0 & 0 & 24,9 \\
\hline Revista da Sociedade Brasileira de M edicina Tropical & 40 & 1,7 & 27,3 & 32 & 1,1 & 22,1 & 48 & 1,1 & 26,0 \\
\hline M emórias do Instituto Oswaldo Cruz & 36 & 1,6 & 28,9 & 47 & 1,6 & 23,7 & 53 & 1,2 & 27,2 \\
\hline Revista Panamericana de Salud Publica & 30 & 1,3 & 30,2 & 39 & 1,3 & 25,0 & 63 & 1,5 & 28,7 \\
\hline Physis & 26 & 1,1 & 31,3 & 21 & 0,7 & 25,7 & 31 & 0,7 & 29,4 \\
\hline Cadernos de Saúde Coletiva (UFRJ) & 23 & 1 & 32,3 & 50 & 1,7 & 27,4 & 69 & 1,6 & 31,0 \\
\hline Jornal de Pediatria & 22 & 1 & 33,3 & 26 & 0,9 & 28,3 & 37 & 0,9 & 31,9 \\
\hline Interface - Comunicação, Saúde, Educação & 22 & 1 & 34,3 & 25 & 0,8 & 29,1 & 46 & 1,1 & 33,0 \\
\hline Transactions of the Royal Society of Tropical M edicine and Hygiene & 20 & 0,9 & 35,2 & 0 & 0 & 29,1 & 15 & 0,3 & 33,3 \\
\hline Epidemiologia e Serviços de saúde (Antigo IESUS) & 18 & 0,8 & 36,0 & 34 & 1,2 & 30,3 & 37 & 0,9 & 34,2 \\
\hline Revista Brasileira de Epidemiologia & 17 & 0,7 & 36,7 & 61 & 2,1 & 32,4 & 82 & 1,9 & 36,1 \\
\hline Revista do Instituto Materno Infantil de Pernambuco & 17 & 0,7 & 37,4 & 0 & 0 & 32,4 & 0 & 0 & 36,1 \\
\hline Revista do Instituto de Medicina Tropical de São Paulo & 16 & 0,7 & 38,1 & 0 & 0 & 32,4 & 24 & 0,6 & 36,7 \\
\hline Saúde em D ebate & 14 & 0,6 & 38,7 & 47 & 1,6 & 34,0 & 17 & 0,4 & 37,1 \\
\hline História, Ciências, Saúde-M anguinhos & 14 & 0,6 & 39,3 & 24 & 0,8 & 34,8 & 21 & 0,5 & 37,6 \\
\hline International Journal of Epidemiology & 14 & 0,6 & 39,9 & 0 & 0 & 34,8 & 0 & 0 & 37,6 \\
\hline Revista Higiene Alimentar & 14 & 0,6 & 40,5 & 0 & 0 & 34,8 & 0 & 0 & 37,6 \\
\hline Arquivos Brasileiros de Cardiologia & 0 & 0 & 0 & 29 & 1 & 35,8 & 27 & 0,6 & 38,2 \\
\hline Revista Brasileira de Educação M édica & 0 & 0 & 0 & 20 & 0,7 & 36,5 & 0 & 0 & 38,2 \\
\hline Brazilian J ournal of M edical and Biological Research & 0 & 0 & 0 & 19 & 0,6 & 37,1 & 25 & 0,6 & 38,8 \\
\hline Revista de Nutrição da PUCCAMP & 0 & 0 & 0 & 19 & 0,6 & 37,7 & 0 & 0 & 38,8 \\
\hline Revista da Associação M édica Brasileira & 0 & 0 & 0 & 18 & 0,6 & 38,3 & 31 & 0,7 & 39,5 \\
\hline São Paulo M edical Journal - Revista Paulista de M edicina & 0 & 0 & 0 & 17 & 0,6 & 38,9 & 0 & 0 & 39,5 \\
\hline Jornal Brasileiro de Pneumologia & 0 & 0 & 0 & 15 & 0,5 & 39,4 & 21 & 0,5 & 40,0 \\
\hline Revista Latino-Americana de Enfermagem (Ribeirão Preto) & 0 & 0 & 0 & 15 & 0,5 & 39,9 & 16 & 0,4 & 40,4 \\
\hline Revista Brasileira de Saúde M aterno Infantil & 0 & 0 & 0 & 0 & 0 & 0 & 46 & 1,1 & 41,5 \\
\hline Revista Baiana de Saúde Pública & 0 & 0 & 0 & 0 & 0 & 0 & 45 & 1 & 42,5 \\
\hline Saúde e Sociedade & 0 & 0 & 0 & 0 & 0 & 0 & 44 & 1 & 43,5 \\
\hline Revista Brasileira de Psiquiatria & 0 & 0 & 0 & 0 & 0 & 0 & 22 & 0,5 & 44,0 \\
\hline Arquivos Brasileiros de Endocrinologia e M etabologia & 0 & 0 & 0 & 0 & 0 & 0 & 22 & 0,5 & 44,5 \\
\hline Lancet & 0 & 0 & 0 & 0 & 0 & 0 & 21 & 0,5 & 45,0 \\
\hline O Mundo da Saúde & 0 & 0 & 0 & 0 & 0 & 0 & 20 & 0,5 & 45,5 \\
\hline Revista Brasileira de Ginecologia e Obstetrícia & 0 & 0 & 0 & 0 & 0 & 0 & 20 & 0,5 & 46,0 \\
\hline Revista de Nutrição & 0 & 0 & 0 & 0 & 0 & 0 & 19 & 0,4 & 46,4 \\
\hline Revista Brasileira de Enfermagem & 0 & 0 & 0 & 0 & 0 & 0 & 17 & 0,4 & 46,8 \\
\hline Revista Brasileira de Nutrição Clínica & 0 & 0 & 0 & 0 & 0 & 0 & 16 & 0,4 & 47,2 \\
\hline Revista Brasileira de Crescimento e Desenvolvimento Humano & 0 & 0 & 0 & 0 & 0 & 0 & 16 & 0,4 & 47,6 \\
\hline AIDS & 0 & 0 & 0 & 0 & 0 & 0 & 15 & 0,3 & 47,9 \\
\hline Diabetes Care & 0 & 0 & 0 & 0 & 0 & 0 & 15 & 0,3 & 48,2 \\
\hline
\end{tabular}


Tabela 5. Classificação das revistas mais utilizadas segundo áreas temáticas.

\begin{tabular}{|c|c|}
\hline Título do periódico & Área temática \\
\hline Revista Brasileira de Educação M édica & Educação médica \\
\hline O M undo da Saúde & Administração hospitalar \\
\hline AIDS & Atenção à saúde \\
\hline Lancet & Atenção à saúde \\
\hline Revista Brasileira de Enfermagem & Atenção à saúde \\
\hline Revista da Associação M édica Brasileira & Atenção à saúde \\
\hline Revista Latino-Americana de Enfermagem (Ribeirão Preto) & Atenção à saúde \\
\hline História, Ciências, Saúde-M anguinhos & Ciências sociais \\
\hline Interface - Comunicação, Saúde, Educação & Ciências sociais \\
\hline Physis & Ciências sociais \\
\hline Saúde e Sociedade & Ciências sociais \\
\hline Arquivos Brasileiros de Cardiologia & Clínicas \\
\hline Diabetes Care & Clínicas \\
\hline Jornal Brasileiro de Pneumologia & Clínicas \\
\hline Jornal de Pediatria & Clínicas \\
\hline Revista Brasileira de Ginecologia e Obstetrícia & Clínicas \\
\hline Revista Brasileira de N utrição Clínica & Clínicas \\
\hline Revista Brasileira de Psiquiatria & Clínicas \\
\hline São Paulo M edical Journal - Revista Paulista de M edicina & Clínicas \\
\hline Arquivos Brasileiros de Endocrinologia e M etabologia & Clínicas \\
\hline Revista de Nutrição & Nutrição e materno-infantil \\
\hline Revista de Nutrição da PU CCAMP & Nutrição e materno-infantil \\
\hline Revista do Instituto Materno Infantil de Pernambuco & Nutrição e materno-infantil \\
\hline Revista Higiene Alimentar & Nutrição e materno-infantil \\
\hline Revista Brasileira de Saúde M aterno-Infantil & Nutrição e materno-infantil \\
\hline Revista Brasileira de Crescimento e Desenvolvimento Humano & Nutrição e materno-infantil \\
\hline Revista da Sociedade Brasileira de M edicina Tropical & Doenças infecciosas e parasitárias \\
\hline Revista do Instituto de M edicina Tropical de São Paulo & Doenças infecciosas e parasitárias \\
\hline Transactions of the Royal Society of Tropical Medicine and Hygiene & Doenças infecciosas e parasitárias \\
\hline Brazilian Journal of M edical and Biological Research & Ciências biológicas \\
\hline M emórias do Instituto Oswaldo Cruz & Ciências biológicas \\
\hline Epidemiologia e Serviços de Saúde (Antigo IESUS) & Epidemiologia \\
\hline International Journal of Epidemiology & Epidemiologia \\
\hline Revista Brasileira de Epidemiologia & Epidemiologia \\
\hline Cadernos de Saúde Coletiva(UFRJ) & Saúde pública \\
\hline Cadernos de Saúde Pública & Saúde pública \\
\hline Ciência \& Saúde Coletiva & Saúde pública \\
\hline Revista Panamericana de Salud Pública & Saúde pública \\
\hline Revista de Saúde Pública & Saúde pública \\
\hline Saúde em Debate & Saúde pública \\
\hline Série Estudos em Saúde Coletiva & Saúde pública \\
\hline Revista Baiana de Saúde Pública & Saúde pública \\
\hline
\end{tabular}

inglês (Tabela 6) . A penas 149 artigos foram escritos em espanhol. A participação de outros idiomas é $1 \%$ a $2 \%$. Não foram incluídos na análise os artigos publicados em 2007. A questão da internacionalização da produção científica éum ponto que merece consideração, dada a importância da cooperação internacional para pesquisa.
Além das principais revistas publicadas no país apresentarem resumos em inglês e, em alguns casos, os próprios artigos serem publicados em inglês, sendo consideradas de circulação internacional (Qualis IA), destaca-se que fazem parte da relação dos periódicos mais utilizados seis revistas estrangeiras: Revista Panamericana 


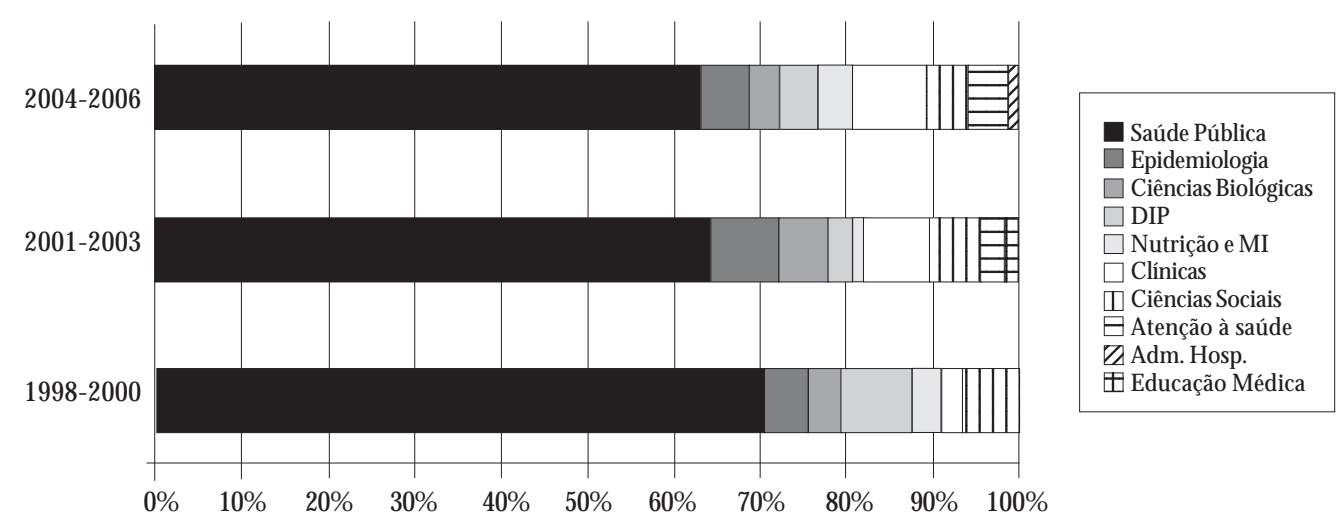

Gráfico 3. Divisão proporcional das áreas temáticas por triênio.

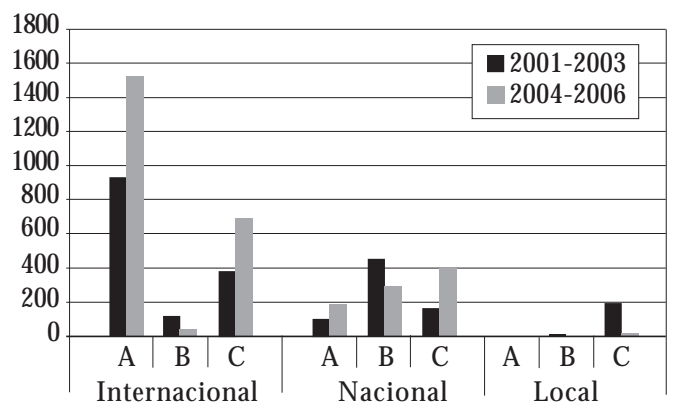

Gráfico 4. Número de artigos publicados em periódicos segundo Qualis.

de Salud Publica, International Journal of Epidemiology, Lancet, Transactions of the Royal Society of Tropical M edicine and Hygiene, AIDS e Diabetes Care.

\section{Autores por artigo}

Analisando-se o número de autores por artigo, observa-seque, en quanto a maioria deles tem apenas um autor, existem dois que foram escritos por mais de 21 autores. Ao longo dos triênios, há uma forte tendência de aumento do número de autores por artigo, como se observa no Gráfico 5. N ote-se que, no terceiro triênio, artigos com dois autores passaram a ser a maioria.
Tabela 6. Numero de artigos segundo idioma de redação por triênio.

\begin{tabular}{|c|c|c|c|c|c|c|c|c|}
\hline \multirow{3}{*}{ Idioma } & \multicolumn{6}{|c|}{ Triênio } & \multirow{2}{*}{\multicolumn{2}{|c|}{$\begin{array}{c}\text { Total } \\
1998-2006\end{array}$}} \\
\hline & \multicolumn{2}{|c|}{$1998-2000$} & \multicolumn{2}{|c|}{ 2001-2003 } & \multicolumn{2}{|c|}{ 2004-2006 } & & \\
\hline & $n$ & $\%$ & $\mathrm{n}$ & $\%$ & $n$ & $\%$ & $\mathrm{n}$ & $\%$ \\
\hline Alemão & 2 & 0,1 & 1 & 0,0 & 2 & 0,0 & 5 & 0,1 \\
\hline Diversos & 1 & 0,0 & 0 & 0,0 & 5 & 0,1 & 6 & 0 \\
\hline Espanhol & 47 & 2,0 & 53 & 1,8 & 49 & 1,1 & 149 & 1,6 \\
\hline Francês & 7 & 0,3 & 11 & 0,4 & 12 & 0,3 & 30 & 0 \\
\hline Inglês & 625 & 27,1 & 873 & 29,6 & 1.194 & 27,8 & 2.692 & 28,2 \\
\hline Italiano & 5 & 0,2 & 1 & 0,0 & 1 & 0,0 & 7 & 0,1 \\
\hline Português & 1.616 & 70,2 & 2.010 & 68,2 & 3.030 & 70,6 & 6.656 & 69,7 \\
\hline Total & 2.303 & 100,0 & 2.949 & 100,0 & 4.293 & 100,0 & 9.545 & 100,0 \\
\hline
\end{tabular}

Distribuição regional

e cooperação entre programas

Quando se analisa a cidade onde está situado o programa, verifica-se que, embora exista uma grande concentração de cursos no Rio de Janeiro e em São Paulo, no terceiro triênio aumenta a participação de programas localizados em cidades da Região N ordeste.

A cooperação entre programas foi analisada levando-se em conta os 1.066 artigos que tiveram a participação de autores de diferentes localidades (Gráfico 6). Do ponto de vista da região ondeestão situados os cursos, a cooperação mais importante (72\%) deu-seentre programas situadosna Região Sudeste. Na Região N ordeste, também ocorreu a participação de mais de um programa na publicação de artigos. 


\section{Conclusões}

Entre os aspectos aqui investigados, o primeiro ponto que chama a atenção éo expressivo crescimento da produção de artigos (completos) publicados em periódicos que respondem por cerca de $60 \%$ da produção científica da área, ao longo dos três triênios analisados. Entretanto, em termos relativos, a evolução do crescimento dos artigos deu-se principalmenteno primeiro (19\% ao ano) e no terceiro triênio ( $12 \%$ ao ano), sen- do que de 2006 para 2007 o sistema registrou um aumento de mais de $30 \%$ na produção de artigos completos em periódicos. Esse acelerado crescimento da produção científica na área foi ressaltado por Barata ${ }^{6,8}$ e Barros ${ }^{7}$ e por vários outros autores em relação à produção nacional de todas as áreas. A produção científica brasileira, em termos relativos, éaque mais cresceu no mundo. Passou de 4.000 artigos indexados em bases internacionais em 1993 (0,7 da produção mundial) para 13.000 em 2004 (1,7\%) e para 30.000 no

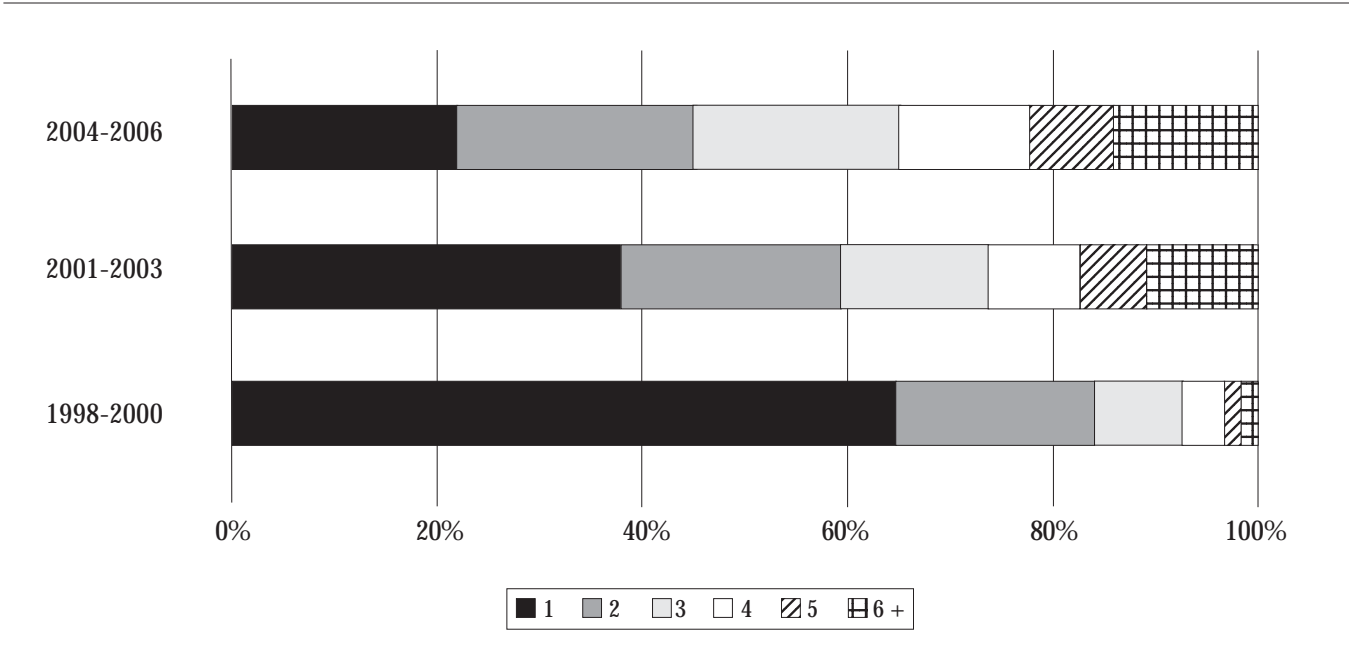

Gráfico 5. Distribuição dos artigos publicados em periódicos segundo o número de autores.

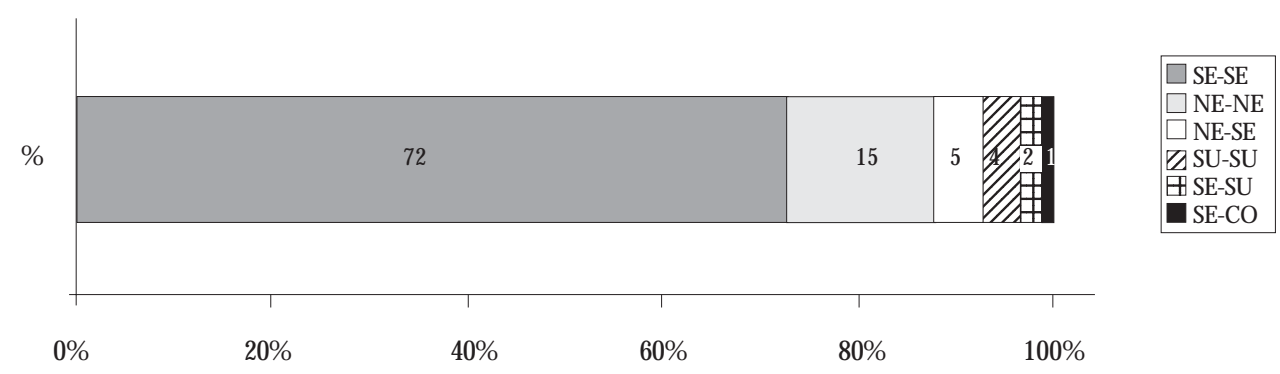

Gráfico 6. Percentual de artigos publicados por dois ou mais programas segundo as regiões geográficas dos programas. 
ano de 2008, passando a representar 2,12\% das publicações mundiais e a ocupar 130 lugar no ranking internacional ${ }^{9}$. Esse incremento ocorreu de forma associada e em sinergia com o aumento do número de cursos de pós-graduação e com 0 aumento exponencial do número de grupos de pesquisa ${ }^{10,12,13}$.

Quanto aos periódicos utilizados para veiculação da produção científica, os autores demonstram preferência por dezenove títulos, nos quais foram publicados pelos menos cinco artigos por ano no período analisado. As revistas mais usadas nos três triênios foram: Cadernos de Saúde Pública (12,1\%), Revista deSaúdePública (6,3\%) eCiência \& SaúdeColetiva (4,8\%). 0 número de títulos cresceu cerca de $20 \%$ entre os triênios ea tendência verificada é de diminuição em termos percentuais de artigos em revistas de saúde pública e aumento da participação em revistas com temáticas mais específicas. N esse sentido, devese destacar o aumento de publicações em revistas de áreas clínicas e de atenção á saúde. Nota-se também uma queda na participação relativa de artigos publicados em revistas com enfoque das ciências sociais nesses triênios. 0 intenso crescimento de artigos submetidos às revistas brasi leiras é uma preocupação dos editores das revistas brasileiras que, como Coimbra ${ }^{14}$, percebem os limites da capacidade de resposta de um sistema de avaliação fortemente apoiado na revisão de pares frente à demanda crescente.

Dados sobre produtividade de artigos publicados em periódicos indicam que, enquanto alguns programas têm tido um crescimento importante ao longo dos triênios, para o conjunto de programas, não se verifica variação entre 0 segundo e o terceiro triênios. Entretanto, a análise foi feita considerando todos os docentes e não apenas os docentes permanentes.

Ao longo do período analisado, houve um expressivo aumento da publicação de artigos em revistas I nternacional A e, em menor proporção, em revistas I nternacional $C, N$ acional $A$ eN acional $C$. N ota-se também uma redução da importância das revistas $\mathrm{N}$ acional $\mathrm{B}$ e locais. Há quese considerar que as três revistas acima mencionadas como as mais usadas são consideradas IA e que apenas $30 \%$ dos artigos foram publicados em inglês, proporção que se manteve nesse patamar nos três triênios.

A exemplo do que severifica em outras áreas, também na Saúde Coletiva, é forte a tendência ao aumento do número de autores por artigo, indicada pela queda na proporção de única autoria, que era de $64 \%$ no primeiro triênio e chegou a $22 \%$ no terceiro triênio, período no qual a proporção com a participação de dois autores passou a ser o modo predominante, com $24 \%$.

Do ponto de vista da expansão dos centros de pós-graduação no país, ainda que Rio de Janeiro e São Paulo sejam as cidades onde se situam a maior parte dos programas e das publicações, já éimportante a produção científica originária de cidades nordestinas, como Fortaleza e Recife. $\mathrm{Na}$ análise dos artigos segundo autoria compartilhada por programas, verifica-se que as maiores cooperações em termos regionais se dão no interior das regi ões Sudeste ( $72 \%$ ) e N ordeste (16\%). A cooperação entre programas do sul e sudeste com programas do nordeste é bem menossignificativa.

Finalmente, é necessário que se façam algumas considerações sobre o sistema COLETA. É nítido ecertamenteimpressionanteo esforço que foi feito no sentido de informatizar todo o sistema que, entretanto, continua apresentando problemas relacionados à falta de críticas na entrada dos dados. Além de detectadas 294 duplicações de registros, a ausência de críticas faz com que, por exemplo, títulos de artigos e de periódicos estejam grafados de diversas maneiras. Isso exige um árduo trabalho de padronização. $\mathrm{N}$ em mesmo em campos mais simples, como o país onde foi obtida a maior titulação do autor, são usadas tabelas padronizadas. 0 sistema também permite que os registros permaneçam incompletos em campos muito importantes para análises das temáticas abordadas, como as palavras-chave definidas pelos autores. A cessão da base de dados para a Abrasco significa um avanço importante na política de acesso às informações da CAPES. Entretanto, a tabulação de dados, às vezes, fica prejudicada pela falta de documentação do sistema, que se resume ao manual do sistema COLETA, do qual constam apenas o dicionário de variáveis e o diagrama de relacionamentos. 


\section{Agradecimentos}

A M argareth Prevot e Rodrigo da Silva M oreira pelo cuidadoso trabalho realizado na organização do banco de dados e na tabulação.

\section{Referências}

1. Paim JS, Teixeira CF. Política, planejamento e gestão em saúde: balanço do estado da arte. Rev. Saude Publica 2006; 40(N Esp):73-78.

2. Nunes ED. A trajetória das ciências sociais em saúde na América Latina: revisão da produção científica. Rev. Saude Publica 2006; 40(N Esp):64-72.

3. Barreto, M. Crescimento e tendência da produção científica em epidemiologia no Brasil. Rev. Saude Publica 2006; 40(N Esp):79-85.

4. Barata RB. A Pós-Graduação e o campo da Saúde Coletiva. Physis 2008; 18(2):189-214.

5. Viacava F, Ramos CL. Difusão da produção científica dos cursos de pós graduação em Saúde Coletiva. Cien Saude Colet 1997; 2(1/2):142-153.

6. Carvalho L, Coimbra JR CEA, Souza-Santos R, Santos RV. Produção e citação em Saúde Coletiva: um olhar a partir dos periódicos. Cad Saude Publica 2007; 23(12):3023-3030.

7. Barata RB. SciELO Saúde Pública: o desempenho dos Cadernos de Saúde Pública e da Revista de Saúde Pública. Cad Saude Publica 2007; 23(12):3031-3040.

8. Barros AJD. Produção científica em saúde coletiva: perfil dos periódicos e avaliação pela Capes. Rev. Saude Publica 2006; 40(N Esp):43-49.

9. M eneghini R. Inusitado aumento da produção científica. Folha de São Paulo 2009; 06 mai.

10. Dagnino R. A comunidade científica e a política de Ciência e Tecnologia: olhando os países avançados. Revista CST 2006; 7(3): 43-58.

11. Guimarães R. Avaliando a avaliação. RBPG 2007; $4(8): 282-292$

12. Martins $C B$, Assad ALD. A pós-graduação e a formação de recursos humanos para inovação. RBPG 1997; 5(1):322-352.

13. Marchelli PS. Formação de doutores no Brasil e no mundo: algumas comparações. RBPG 2005; 2(3):729.

14. Coimbra CEA. Efeitos colaterais do produtivismo acadêmico na pós-graduação. Cad Saude Publica 2009; 25(10):2092-2093.

Artigo apresentado em 07/12/2009

Aprovado em 12/01/2010

Versão final apresentada em 19/02/2010 